\title{
Evolution and innovations in the systemic treatment landscape of renal cell carcinoma
}

\author{
Alessia Mennitto ${ }^{1}$, Elena Verzoni ${ }^{1}$, Giuseppe Procopio $^{1}$
}

\begin{abstract}
Treatments for metastatic renal cell carcinoma are constantly changing thanks to the increasing knowledge of the molecular biology of this tumor. In fact, understanding the underlying genetic mechanisms has led to the development of new classes of drugs, known as molecularly targeted therapies that, to date, represent the standard treatment in the first-line setting. More recently, we have witnessed the resurgence of immunotherapy, which is providing significant benefit to patients with treatment-refractory disease. The use of all of the currently available agents, in sequence, has been shown to prolong survival to beyond 20 months, and to improve the quality of life of patients. Despite the recent progress, there is still a crucial need for further advances. The discovery of biomarkers that would allow the prediction of treatment response and therefore lead to better patient selection, as well as understanding the potential mechanisms of acquired resistance that might guide the choice of the optimal sequential use of the available drugs, together still represent an open challenge for both scientists and clinicians.
\end{abstract}

Key words: renal cell carcinoma, targeted cancer therapies, immunotherapy

\section{Introduction}

Renal cell carcinoma (RCC) represents about 3\% of all malignant tumors and, after prostate and bladder cancer, is the third most common urologic neoplasm [1]. The number of new cases per year has increased worldwide from 1975 [1]; part of this rise probably correlates with the increasing detection of localized and asymptomatic tumors by imaging procedures, but this cannot fully explain the increased incidence of advanced RCC and mortality. Therefore, other factors may be contributing to the described phenomenon.

Great strides have been made in the treatment of metastatic RCC (mRCC) allowing more patients to live with the disease and maintain their normal lifestyle. For many years, interleukin- 2 and interferon- $\alpha$ (IFN- $\alpha$ ) have had a dominant role notwithstanding temporary responses and relevant toxicity [2]. The advent of drugs targeting the vascular endothelial growth factor (VEGF) and mammalian target of rapamycin (mTOR) pathways has completely changed the outcome of metastatic patients, improving progression-free survival (PFS), overall survival (OS) and quality of life [3, 4]. The best sequence of their use as targeted agents is not yet clear, while their combinations have shown high toxicity without improvement of the prognosis of patients. More recently, the introduction of the immune-checkpoint inhibitors has further expanded the landscape in the treatment of advanced RCC.

\section{First-line treatments (Table 1)}

Based on the results of two phase III trials [5, 6], firstline treatment options for patients with low or intermediate risk clear cell mRCC are represented by sunitinib, an oral multitargeted receptor tyrosine kinase inhibitor (TKI), and pazopanib, a multikinase angiogenesis inhibitor. A direct comparative study, the COMPARZ trial [7], showed similar effectiveness in response (31\% pazopanib vs 25\% sunitinib), PFS (pazopanib 8.4 months, sunitinib 9.5 months) and OS (pazopanib 28.4 months, sunitinib 29.3 months, $p=0.275$ ), with slight differences in terms of toxicity. Fatigue, hand-foot syndrome, sto-

'Genitourinary Unit, Medical Oncology Department, Fondazione IRCCS Istituto Nazionale dei Tumori, Milan, Italy. Correspondence to:

Giuseppe Procopio,

Medical Oncology Unit 1,

Fondazione IRCCS Istituto Nazionale dei Tumori,

Via Venezian 1, 20133 Milano, Italy.

Phone: +390223904450 - Fax: +390223902149

E-mail: giuseppe.procopio@istitutotumori.mi.it

CANCER BREAKING NEWS 2017;5(1):7-11

DOI: $10.19156 / c b n .2017 .0032$ 
Table 1. First-line trials in metastatic renal cell carcinoma.

\begin{tabular}{|c|c|c|c|c|}
\hline \multirow{2}{*}{ Regimen } & \multicolumn{3}{|c|}{ Results } & \multirow{2}{*}{ References } \\
\hline & mPFS (mo.) & mOS (mo.) & ORR (\%) & \\
\hline Sunitinib $v s$ IFN- $\alpha$ & 11 vs 5 & 26.4 vs 21.8 & 31 vs 6 & Motzer 2007 [5] \\
\hline Pazopanib vs Placebo & $9.2 v s 4.2$ & 22.9 vs 20.5 & $30 v s 3$ & Sternberg 2010 [6] \\
\hline Sunitinib vs Pazopanib & 9.5 vs 8.4 & 29.1 vs 28.3 & 25 vs 31 & Motzer 2013 [7] \\
\hline Bevacizumab-IFN- $\alpha$ vs IFN- $\alpha$ & 10.2 vs 5.4 & 23.3 vs 21.3 & 31 vs 13 & Escudier 2007 [9] \\
\hline Temsirolimus $v s$ Temsirolimus-IFN- $\alpha$ vs IFN- $\alpha$ & $5.5 v s 4.7 v s 3.1$ & 10.9 vs 8.4 vs 7.3 & 8.6 vs 8.1 vs 4.8 & Hudes 2007 [10] \\
\hline Sunitinib vs Cabozantinib & 5.6 vs 8.2 & not reached & $18 v s 46$ & Choueiri 2017 [11] \\
\hline
\end{tabular}

IFN: interferon; mo.: months; mOS: median overall survival; mPFS: median progression-free survival; ORR: objective response rate.

matitis and myelosuppression were more frequent with sunitinib, whereas liver toxicity and hair color changes were more common with pazopanib. When patient preference was assessed in the phase III PISCES trial, 70\% of patients preferred pazopanib due to a better quality of life, compared with the $22 \%$ of patients who preferred sunitinib [8].

Despite having been approved for first-line treatment in patients with a low or intermediate risk profile, the combination of bevacizumab, a recombinant anti-VEGF monoclonal antibody, and IFN- $\alpha$, is used less and less in everyday clinical practice, due to lower activity and higher toxicity [9].

In poor-risk mRCC, temsirolimus, an mTOR inhibitor, has the highest evidence as a first-line therapy. In a randomized phase III trial, it has shown its superiority over IFN- $\alpha$ in terms of OS (median OS $10.9 v s 7.3$ months) and PFS [10].

A recently published phase II trial (CABOSUN) compared sunitinib versus cabozantinib, a multikinase inhibitor, as front-line targeted therapy. This study showed the superiority of cabozantinib in terms of PFS (8.2 vs 5.6 months, $\mathrm{p}=0.012)$ and ORR ( $46 \%$ vs $18 \%)$ in patients with untreated $\mathrm{mRCC}$ of intermediate or poor risk, compared to sunitinib [11].

\section{Second and subsequent} treatment lines (Table 2)

After progression on bevacizumab or TKI therapy, multiple agents are available in the second-line setting.

Sorafenib, a dual-action inhibitor that targets RAF/MEK/ ERK and VEGF receptor (VEGFR)/platelet-derived growth factor receptor (PDGFR) pathways, was the first TKI that, in 2005, was approved by the US Food and Drug Administration (FDA) in the second-line setting [12].

Axitinib, a potent inhibitor of VEGFRs, was approved after the results of the phase III trial AXIS, in which it prolonged PFS by two months more than sorafenib (6.7 vs 4.7 months, hazard ratio [HR] 0.665) and obtained a higher objective response rate (ORR): $19.4 \%$ vs $9.4 \%$ [13]. The axitinib arm was also better tolerated with less discontinuation of therapy.

The phase III RECORD-1 trial provided support for the use of the mTOR inhibitor everolimus. In this study, everolimus was associated with a doubling of PFS compared with placebo (4.0 vs 1.9 months) in patients pretreated with sunitinib, sorafenib or both $[4,14]$.

The renewed interest in cancer immunotherapies over recent years has led to the development of a new class of treatments, the T-cell checkpoint inhibitors, such as nivolumab [15]. Based on the randomized phase III trial

Table 2. Second-line and beyond trials in advanced renal cell carcinoma.

\begin{tabular}{|c|c|c|c|c|}
\hline \multirow{2}{*}{ Regimen } & \multicolumn{3}{|c|}{ Results } & \multirow{2}{*}{ References } \\
\hline & mPFS (mo.) & mOS (mo.) & ORR (\%) & \\
\hline Axitinib vs Sorafenib & $6.7 v s 4.7$ & 20.1 vs 19.2 & $19 v s 9$ & Rini 2011 [18] \\
\hline \multirow[t]{2}{*}{ Everolimus vs Placebo } & 4.9 vs 1.9 & $14.8 v s 14.4$ & $1.8 v s 0$ & Motzer 2008 [4] \\
\hline & & & & Motzer 2010 [14] \\
\hline Nivolumab vs Everolimus & $4.6 v s 4.4$ & 25 vs 19.6 & 25 vs 5 & Motzer 2015 [16] \\
\hline \multirow[t]{2}{*}{ Cabozantinib vs Everolimus } & 7.4 vs 3.9 & 21.4 vs 16.5 & 17 vs 3 & Choueiri 2015 [20] \\
\hline & & & & Choueiri 2016 [21] \\
\hline Lenvatinib-Everolimus vs Lenvatinib vs Everolimus & $12.8 v s 9$ vs 5.6 & 25.5 vs 19.1 vs 15.4 & 35 vs 39 vs 0 & Motzer 2015 [26] \\
\hline
\end{tabular}

mo.: months; mOS: median overall survival; mPFS: median progression-free survival; ORR: objective response rate. 
CheckMate 025, nivolumab, an antibody to the programmed cell death-1 (PD-1) receptor, gained approval in $\mathrm{mRCC}$ patients pre-treated with at least one prior antiangiogenic therapy. In the trial above, nivolumab was compared with everolimus, showing an improved OS (25 vs 19.6 months, HR 0.73) and ORR (25\% vs 5\%, $\mathrm{p}<0.001)$ with a lower incidence of adverse events and a better quality of life $[16,17]$. Based on the results of this study, nivolumab was approved in patients with $\mathrm{mRCC}$ after prior antiangiogenic therapy.

Eventually, all patient become resistant to single-agent VEGF inhibitors. The mechanisms of this acquired resistance are not completely understood. Genetic modifications or the up-regulation of the target proteins and the activation of alternative non-VEGF pathways promoting angiogenesis, cell proliferation, and invasion may play an important role. In particular, preclinical studies have identified MET, AXL and FGFR signaling pathways among the factors implicated in the pathogenesis of RCC and affecting the development of resistance [18].

In this landscape, several preclinical and clinical studies have investigated the activity and safety of cabozantinib, an inhibitor of multiple TKI receptors, including MET, VEGFRs, and AXL [19]. A phase III trial (METEOR) was designed to investigate the superiority of cabozantinib over everolimus in patients who progressed after antiangiogenic therapies [20, 21]. Cabozantinib improved PFS (7.4 vs 3.8 months), OS (HR 0.67), and ORR ( $21 \%$ vs $5 \%$ ) compared with everolimus. The spectrum of side effects in the cabozantinib arm was similar to the other VEGF inhibitors (hypertension, nausea, diarrhea, fatigue, loss of appetite, and hand-foot syndrome), on the other hand, there was more anemia, pneumonitis, peripheral edema and hyperglycemia with everolimus. Cabozantinib required more dose reductions $(60 \%$ vs $25 \%$ with everolimus); notwithstanding this, the rate of discontinuation was similar in the two arms ( $9 \%$ vs $10 \%$, respectively). The results of this study led to the approval by the US FDA in April 2016 of cabozantinib after a previous antiangiogenic therapy.

Considering that the FDA has approved both cabozantinib and nivolumab for the same indications, we are not sure on where to place them in the treatment algorithm. Perhaps, following nivolumab with cabozantinib might represent a reasonable choice for patients pretreated with a TKI [22].

Other strategies tested in order to overcome acquired resistances were the combinations of VEGF and mTOR inhibitors. The couplets bevacizumab plus temsirolimus or everolimus compared with bevacizumab plus IFN- $\alpha$ did not provide greater efficacy, at the cost of increased toxicity [23-25]. Conversely, in a phase II trial, the combination of everolimus with lenvatinib, which targets both the VEGF and the fibroblast growth factor (FGF) pathway, prolonged PFS (14.5 vs 5.5 months; $\mathrm{p}=0.0005$ ) when compared with everolimus alone. The toxicity profile, characterized by asthenia, loss of appetite and diarrhea, was manageable with dose reductions, which occurred in $71 \%$ of patients [26]. On the basis of these results, in May 2016 the combination of lenvatinib and everolimus gained FDA approval for $\mathrm{mRCC}$ following one prior VEGF-TKI.

From the third line onwards, treatments are not standardized, because much of our knowledge derives from retrospective analyses that have also shown a survival advantage with targeted agents in advanced lines, mostly in patients with favorable- and intermediate-prognostic criteria disease. The best sequence is still debated; some prospective trials [27, 28] and retrospective analyses [29] are not conclusive in preferring one sequence rather than another, and this represents an open issue that would require a large prospective randomized trial to clarify.

\section{Looking ahead}

Current research is working on several fronts, such as the use of known drugs for different indications, combinatorial approaches, cancer vaccines and the development of novel classes of immune checkpoint inhibitors, as well as new targeted agents.

Combination therapy strategies are currently being used in the investigation of associations between different immune checkpoint blockers (e.g. nivolumab-ipilimumab, an anti-CTLA4, NCT02231749), anti-VEGF therapy and immunotherapy, such as bevacizumab with atezolizumab (NCT01984242) or nivolumab (NCT02210117), pembrolizumab plus aflibercept [30], pazopanib (NCT02014636), axitinib (NCT02133742) or lenvatinib (NCT02501096), and avelumab with axitinib (NCT02493751).

At present, many VEGF, PDGF, and FGF inhibitors are under investigation, including regorafenib, tivozanib, cediranib, linifanib, dovitinib, and brivanib. Moreover, novel classes of drugs with new mechanisms of actions, e.g. inhibitors of MET, PI3K, Akt, TORC2, Ang-1 and Ang-2, are currently being studied in early clinical trials. Finally, new tumor vaccines are under evaluation, alone or in combinatorial approaches, in the context of the immune modulation strategies (NCT01582672, NCT01265901).

\section{Conclusions}

In recent years, therapeutic options for renal cancer have rapidly expanded, and they are destined to further evolve 
in the coming years. The treatment landscape is increasingly crowded, primarily in the second line. Thus further clinical trials are needed in order to compare the new agents head-to-head and allow an algorithm for the optimal treatment of mRCC to be designed.

Currently, reliable biomarkers that could predict the success or failure of therapies and allow personalized medicine are an ongoing field of research. Thus, the selection by the clinician of one agent rather than another and the choice of the best sequence of treatment is based on individual patient characteristics and medical history, as well as the safety profile of the different drugs, with the aim of prolonging the survival of patients and maintaining a good quality of life.

\section{Acknowledgments}

The authors thank Ray Hill, an independent medical writer, who provided native English editing and journal styling on behalf of HPS. This editorial assistance was funded by PharmaMar, Spain.

\section{Conflicts of Interest}

The Authors declare there are no conflicts of interest in relation to this article.

\section{References}

1. European Network of Cancer Registries. Eurocim version 4.0. European incidence database V2.3, 730 entity dictionary (2001), Lyon, France, 2001.

2. Coppin C, Porzsolt F, Autenrieth M et al. Immunotherapy for advanced renal cell cancer. Cochrane Database Syst Rev 2015(12):CD001425.

3. Iacovelli R, Sternberg CN, Porta $\mathrm{C}$ et al. Inhibition of the VEGF/VEGFR pathway improves survival in advanced kidney cancer: a systematic review and meta-analysis. Curr Drug Targets 2015;16(2):164-70.

4. Motzer RJ, Escudier B, Oudard S et al.; RECORD-1 Study Group. Efficacy of everolimus in advanced renal cell carcinoma: a double-blind, randomised, placebo-controlled phase III trial. Lancet 2008;372(9637):449-56.

5. Motzer RJ, Hutson TE, Tomczak P et al. Sunitinib versus interferon alfa in metastatic renal-cell carcinoma. N Engl J Med 2007;356(2):115-24.

6. Sternberg CN, Davis ID, Mardiak J et al. Pazopanib in locally advanced or metastatic renal cell carcinoma: results of a randomized phase III trial. J Clin Oncol 2010;28(6):1061-8.

7. Motzer RJ, Hutson TE, Cella D et al. Pazopanib versus sunitinib in metastatic renal-cell carcinoma. N Engl J Med 2013;369(8):722-31.

8. Escudier B, Porta C, Bono P et al. Randomized, controlled, double-blind, cross-over trial assessing treatment preference for pazopanib versus sunitinib in patients with metastatic renal cell carcinoma: PISCES Study. J Clin Oncol 2014;32(14):1412-8.

9. Escudier B, Pluzanska A, Koralewski P et al.; AVOREN Trial Investigators. Bevacizumab plus interferon alfa-2a for treatment of metastatic renal cell carcinoma: a randomised, double-blind phase III trial. Lancet 2007;370(9605):2103-11.

10. Hudes G, Carducci M, Tomczak P et al.; Global ARCC Trial. Temsirolimus, interferon alfa, or both for advanced renal-cell carcinoma. N Engl J Med 2007;356(22):2271-81.

11. Choueiri TK, Halabi S, Sanford BL et al. Cabozantinib versus sunitinib as initial targeted therapy for patients with metastatic renal cell carcinoma of poor or intermediate risk: the Alliance A031203 CABOSUN trial. J Clin Oncol 2017;35(6):591-7.

12. Escudier B, Eisen T, Stadler WM et al.; TARGET Study Group. Sorafenib in advanced clear-cell renal-cell carcinoma. N Engl J Med 2007;356(2):125-34.

13. Rini BI, Escudier B, Tomczak P et al. Comparative effectiveness of axitinib versus sorafenib in advanced renal cell carcinoma (AXIS): a randomised phase 3 trial. Lancet 2011;378(9807):1931-9.

14. Motzer RJ, Escudier B, Oudard S et al.; RECORD-1 Study Group. Phase 3 trial of everolimus for metastatic renal cell carcinoma: final results and analysis of prognostic factors. Cancer 2010;116(18):4256-65.

15. Mennitto A, Grassi P, Ratta R et al. Nivolumab in the treatment of advanced renal cell carcinoma: clinical trial evidence and experience. Ther Adv Urol 2016;8(5):319-26.

16. Motzer RJ, Escudier B, McDermott DF et al.; CheckMate 025 Investigators. Nivolumab versus everolimus in advanced renal-cell carcinoma. N Engl J Med 2015;373(19):1803-13.

17. Cella D, Grünwald V, Nathan P et al. Quality of life in patients with advanced renal cell carcinoma given nivolumab versus everolimus in CheckMate 025: a randomised, openlabel, phase 3 trial. Lancet Oncol 2016;17(7):994-1003.

18. Rini BI, Atkins MB. Resistance to targeted therapy in renalcell carcinoma. Lancet Oncol 2009;10(10):992-1000.

19. Grassi P, Verzoni E, Ratta R et. Cabozantinib in the treatment of advanced renal cell carcinoma: design, development, and potential place in the therapy. Drug Des Devel Ther 2016;10:2167-72.

20. Choueiri TK, Escudier B, Powles T et al.; METEOR Investigators. Cabozantinib versus everolimus in advanced renalcell carcinoma. N Engl J Med 2015;373(19):1814-23.

21. Choueiri TK, Escudier B, Powles T et al.; METEOR Investigators. Cabozantinib versus everolimus in advanced renal cell carcinoma (METEOR): final results from a randomised, open label, phase 3 trial. Lancet Oncol 2016;17(7):917-27.

22. Procopio G, Ratta R, Grassi P. Evolving treatment landscape in metastatic renal cell carcinoma: where are we now? Expert Rev Anticancer Ther 2016;16(2):133-5.

23. Négrier S, Gravis G, Pérol D et al. Temsirolimus and bevacizumab, or sunitinib, or interferon alfa and bevacizumab for patients with advanced renal cell carcinoma (TORAVA): a 
randomized phase 2 trial. Lancet Oncol 2011;12(7):673-80.

24. Ravaud A, Barrios CH, Alekseev B et al. RECORD-2: phase II randomized study of everolimus and bevacizumab versus interferon $\alpha-2 \mathrm{a}$ and bevacizumab as first-line therapy in patients with metastatic renal cell carcinoma. Ann Oncol 2015;26(2):1378-84.

25. Rini BI, Bellmunt J, Clancy J et al. Randomized phase III trial of temsirolimus and bevacizumab versus interferon alfa and bevacizumab in metastatic renal cell carcinoma: INTORACT trial. J Clin Oncol 2014;32(8):752-9.

26. Motzer RJ, Hutson TE, Glen H et al. Lenvatinib, everolimus, and the combination in patients with metastatic renal cell carcinoma: a randomised, phase 2, open-label, multicentre trial. Lancet Oncol 2015;16(15):1473-82.

27. Hutson TE, Escudier B, Esteban E et al. Randomized phase III trial of temsirolimus versus sorafenib as second-line therapy after sunitinib in patients with metastatic renal cell carcinoma. J Clin Oncol 2014;32(8):760-7.

28. Eichelberg C, Vervenne WL, De Santis M et al. SWITCH: a randomised, sequential, open-label study to evaluate the efficacy and safety of sorafenib-sunitinib versus sunitinibsorafenib in the treatment of metastatic renal cell cancer. Eur Urol 2015;68(5):837-47.

29. Iacovelli R, Cartenì G, Sternberg $\mathrm{CN}$ et al. Clinical outcomes in patients receiving three lines of targeted therapy for metastatic renal cell carcinoma: results from a large patient cohort. Eur J Cancer 2013;49(9):2134-42.

30. Dudek A, Sica R, Sidani A et al. Phase Ib study of pembrolizumab in combination with bevacizumab for the treatment of metastatic renal cell carcinoma: Big Ten Cancer Research Consortium BTCRC-GU14-003 [Abstract]. J Clin Oncol 2016;34(Suppl 2S; abstr 559). 